Citation: J Holmes, J Phillips, R Morris, University of Nottingham, Y Bedekar, Independent Occupational Therapist, $R$ Tyerman, Buckinghamshire Healthcare Trust, $K$ Radford, University of Nottingham. British Journal of Occupational Therapy - Manuscript ID 212-Nov-2015-RP.R2. In Press

\title{
Development and evaluation of an early specialised traumatic brain injury vocational rehabilitation training package.
}

\section{Short Title:}

Training OTs to deliver vocational rehabilitation to people with traumatic brain injury.

\section{Authors:}

Jain Holmes, Dr Julie Phillips, Richard Morris, Ms Yashashree Bedekar, Mrs Ruth Tyerman, Dr Kate Radford.

\section{Contact Details of Authors:}

Jain Holmes (corresponding author),

UKOTRF and UoN PhD Research Fellow, University of Nottingham

Division of Rehabilitation and Ageing, School of Medicine, B109 Medical School, Queens Medical Centre, Nottingham, NG7 2UH

$+44(0) 1158230480$

jain.holmes@nottingham.ac.uk

No competing interests.

Dr Julie Phillips.

Research Occupational Therapist, University of Nottingham

No competing interests.

Mr Richard Morris

Research Assistant, University of Nottingham

No competing interests. 
Ms Yashashree Bedekar

Independent Occupational Therapist

No competing interests.

Mrs Ruth Tyerman, Buckinghamshire Healthcare Trust

Programme Manager

No competing interests.

Dr Kathryn Radford, University of Nottingham

Associate Professor in Rehabilitation Research (Long Term Conditions)

No competing interests.

The Authors declare that there is no conflict of interest.

\section{Ethics approval:}

1. NRES Committee East Midlands - Northampton, 21/10/2013, ref: 13/EM/0353

2. School of Medicine Rehabilitation and Ageing, University of Nottingham, 19/12/2013 (REC ref: 13/EM/0353), D14112013 FRESH

\section{Trial Details:}

The Facilitating return to work through early specialist health-based interventions (FRESH) trial registered ISRCTN Number is 38581822 . This study is sponsored by the University of Nottingham, Research and Graduate Services, Kings Meadow Campus, Lenton Lane, Nottingham, NG7 2NR, UK.

This project is funded by the NIHR HTA Programme (project number 11/66/02). The views and opinions expressed herein are those of the authors and do not necessarily reflect those of the HTA programme, NIHR, NHS or the Department of Health.

http://www.isrctn.com/ISRCTN38581822 


\section{Trial website:}

\section{http://www.nottingham.ac.uk/go/fresh}

\section{Funding}

This project was funded by the National Institute for Health Research HTA Programme [project number 11/66/02] The views and opinions expressed therein are those of the authors and do not necessarily reflect those of HTA, NIHR, NHS or the Department of Health; PhD funding for J Holmes provided by UK Occupational Therapy Research Fund and University of Nottingham.

\section{Acknowledgements}

The first author would like to acknowledge the commitment of the training team in the development and delivery of the package. Additionally the funders are acknowledged for their financial support of the study.

\section{Development and evaluation of an early specialised traumatic brain injury vocational rehabilitation training package.}

\section{Abstract}

Background: In a feasibility randomised controlled trial, training was developed to equip Occupational Therapists to deliver Early Specialised Traumatic Brain Injury Vocational Rehabilitation in the English National Health Service.

Methods: The package was developed by "experts" in vocational rehabilitation and traumatic brain injury and included a manual, direct instruction by six trainers and opportunity for mentorship by four therapists. Following training, therapists were interviewed regarding the effectiveness and "ease of use" of the package. Interviews were analysed using the framework approach.

Results: Five trained therapists were interviewed regarding the package. Results were organised into 6 categories: (1) motivation to participate in research; (2) impact of the learning environment; (3) changing confidence levels over time; (4) growing appreciation of complexities 
about the intervention; (5) acceptability of the training package; (6) lessons for future implementation.

Conclusions: Therapists reported acquiring knowledge necessary to implement the intervention. Data indicates that training packages require detailed descriptions of the interventions being taught for local implementation in the NHS and for future research. Training materials are valued by therapists but require time for familiarisation and reminders from mentors help put training into practice. Therapists have concerns about implementing interventions within a research context, which researchers should address.

Keywords: Vocational rehabilitation, traumatic brain injury, implementation

\section{Introduction}

TBI is a major cause of long term disability (Saltychev et al., 2013). People who incur severe TBI are likely to sustain permanent neurological damage, which has a lasting impact on the ability to resume former occupations. Whilst a proportion of those with milder injury recover and return to work (RTW) (Cancelliere et al., 2014), some continue to experience physical, cognitive and psychosocial symptoms (Cancelliere et al., 2013), which limit participation in social and vocational activities (Cancelliere et al., 2014).

Enabling people who have capacity to work to do so is a UK Government priority (Department for Work and Pensions, 2013) a recognised role for healthcare professionals (Black and Frost, 2011); and a National Health Service (NHS) outcome (Department of Health, 2010). Vocational Rehabilitation (VR) is defined as, "a multiprofessional approach that is provided to individuals of working age with health-related impairments, limitations, or restrictions with work functioning and whose primary aim is to optimise work participation" (Escorpizo et al., 2011). In the UK health system rehabilitation commissioning is patchy resulting in the delivery of VR meeting less than $10 \%$ of the estimated need for people with TBI (Playford et al., 2011). 
VR is a complex intervention with several interacting components (Medical Research Council, 2008). It is difficult to standardise and best delivered in a personalised way which is sensitive to local contexts. VR targets not only the brain injured person but also their family, the work environment and the employer; thus crossing health, employment, social care and welfare contexts. Implementing such a complex intervention into complex contexts is not straightforward (Damschroder et al., 2009).

Health interventions should be clearly described and include the elements of complexity that affect its implementation (Richards DA, 2015). One aspect of implementation is how effectively practitioners learn about interventions and another that clear descriptions of interventions within training are important. To improve the standards of reporting about training packages, the Workgroup for Intervention Development and Evaluation Research (WIDER) has developed guidance for their descriptions (Albrecht et al., 2013).

The specific VR used in this study was initially delivered by the Nottingham TBI Service (NTBIS); Early Specialist TBI VR (ESTVR). It is novel in the NHS because it is delivered very early after brain injury and specifically aims to keep people in work. ESTVR has been previously evaluated and described (Phillips, 2013; Phillips et al., 2010; Radford et al., 2013a). It is based on best practice recommendations for VR (The Royal College of Physicians and The British Society of Rehabilitation Medicine, 2003) and adopts a case coordination model (Fadyl and McPherson, 2009) where an OT and case manager deliver ESTVR. This intervention is being studied in the NIHR-funded multi-centre feasibility randomised controlled trial FRESH (Facilitating RTW through Early Specialist Health-based interventions (Radford et al., 2013b)). Occupational Therapists (OTs) employed or seconded onto the trial needed to learn how to deliver the complex ESTVR intervention over its 12-month duration with each participant. We used data from the FRESH trial to answer the research questions detailed in this study. 
The purpose of the study was to explore the following questions: (1) Could the complex intervention, ESTVR, be translated into a training manual, direct teaching and mentoring package for OTs working in the FRESH trial?; (2) Did the OTs find the training package effective in preparing them to deliver the intervention?; (3) How did the OTs describe the package's "ease of use"?

\section{Method}

A training package was developed for OTs employed to deliver ESTVR in the FRESH trial. It included direct teaching, a manual and mentoring. Table 1 provides an overview of the process. The WIDER guidelines (Albrecht et al., 2013) are used to provide a detailed description of the training package.

Table 1. Overview of the ESTVR training development and delivery process.

\begin{tabular}{|l|l|l|l|l|l|}
\hline March 2013 & $\begin{array}{l}\text { September } \\
2013\end{array}$ & October 2013 & April 2014 & October 2014 & October 2015 \\
\hline $\begin{array}{l}\text { Training } \\
\text { package } \\
\text { development }\end{array}$ & $\begin{array}{l}\text { Training } \\
\text { delivered to } \\
\text { all OTs }\end{array}$ & $\begin{array}{l}\text { FRESH trial } \\
\text { starts } \\
\text { recruitment }\end{array}$ & $\begin{array}{l}\text { Refresher } \\
\text { training } \\
\text { delivered to } \\
\text { all OTs }\end{array}$ & $\begin{array}{l}\text { FRESH trial } \\
\text { stops } \\
\text { recruitment }\end{array}$ & $\begin{array}{l}\text { FRESH trial } \\
\text { ends }\end{array}$ \\
\cline { 2 - 4 } & OTs deliver ESTVR \\
\cline { 2 - 5 } Mentoring provided \\
\cline { 2 - 4 }
\end{tabular}

\section{Development of the manual}

An 'expert' training group was convened with members identified for their expertise in VR, TBI and training delivery and included a service-user. Three development meetings were held, supplemented by email and telephone communication. The existing NTBIS manual and a pro forma used to describe the content of ESTVR (Phillips et al., 2010) were used as starting points of the manual development. The NTBIS OT was questioned about her experiential knowledge until the training group was satisfied that they understood the intervention being delivered and could describe its content and process. 
Literature and clinical guidelines on TBI and RTW were collated and integrated into the manual (College of Occupational Therapists, no date-a; College of Occupational Therapists, no date-b; das Nair et al., 2012; Phillips, 2013; Playford and Sweetland, 2010); Examples of manuals developed for other trials of rehabilitation were used to inform the design of the manual (das Nair et al., 2012; Garfield et al., 2013; Logan et al., 2014; Walker et al., 2012).

From this knowledge a draft manual was created and reviewed multiple times until the group agreed upon the end product. The final output was a manual, produced in hard and soft copy, which was issued to each OT. In order to optimise local implementation, the OTs were encouraged to fit the manual to their context by adding additional information e.g., local contact details for Jobcentre Plus, Headway groups etc.

\section{Manual Content}

The introduction explained the issues surrounding TBI and RTW. The first five chapters followed a typical patient journey from hospital admission to RTW. Chapters are detailed in Table 2.

Table 2. ESTVR Manual

\begin{tabular}{|l|l|}
\hline Chapter & Content \\
\hline One & Initial work preparation prior to RTW \\
\hline Two & Graded RTW process \\
\hline Three & Job retention support \\
\hline Four & Alternative occupation options if returning to a former job was not appropriate \\
\hline Five & Discharge process from ESTVR \\
\hline Six & Supporting documentation to deliver ESTVR \\
\hline Seven & References and resources related to TBI and RTW \\
\hline Eight & Templates for paperwork (letter examples, information leaflets about TBI \\
\hline
\end{tabular}




\begin{tabular}{|l|l|}
\hline & symptoms) \\
\hline Nine & Relevant RTW research for therapists, employers and people with TBI \\
\hline
\end{tabular}

Chapters two to five had the same structure covering; the aims of ESTVR; examples of specific intervention activities; the role of the OT and Case Manager; information on the frequency of visits and a section on common TBI issues related to RTW.

Interviews with the OTs were used to evaluate the manual and are described below. Once the manual was developed, methods to transfer this knowledge (the direct instruction and mentoring) progressed.

\section{Development, delivery and evaluation of the direct instruction}

A multi-modal approach blending didactic teaching with case vignette discussions and role play was chosen. This was considered the optimum strategy to achieve learning (Petzold et al., 2010). Training was scheduled to take place within a month of trial recruitment to ensure learning remained current. However, trial recruitment was delayed by three months in two centres and six months in the third. Refresher training was scheduled six months after the initial training to provide peer support once OTs had case experience.

Training was delivered by training group members, NTBIS case managers and former service users. A two-day training session was considered appropriate bearing in mind difficulties being released from busy workloads in the NHS. It was intended that all OTs were taught together to provide peer support.

As the OTs were not already in post when the training was developed, assumptions were made that each would have some knowledge of TBI but little knowledge of VR. As such, pre-training reading materials were sent to each therapist so they arrived for training with a common understanding of VR. Additionally a learning needs analysis (LNA) was developed to ascertain 
the therapists' existing knowledge and confidence on TBI and VR, [using a scale of (1) not very confident or knowledgeable to (5) already really confident and knowledgeable]. This was emailed to each OT one month before the training to allow time to incorporate identified needs into the direct instruction.

The manual and hard copies of presentations were issued during the training. For contingency planning, training was video-recorded using the University's Echo 360 service and made available to all OTs as a resource. At the end of day one homework (case study problemsolving) was provided to embed learning.

In order to evaluate training effectiveness and enable further development of the training programme, OTs were asked to assess their own knowledge of and ability to deliver ESTVR before and after training using a questionnaire developed by (O'Brien et al., 2013) and adapted for use in this study. O'Brien's questionnaire sought the perceptions of OTs, working in rheumatology, who were engaged in a VR training programme. OTs in this study answered questions related to their knowledge about key aspects of TBI and their confidence to deliver ESTVR (Table 3) [using a scale of poor (1) to very good (5)]. They also answered open-ended questions about aspects of the programme and were asked for suggestions for improvements to training.

\section{Development and evaluation of mentoring}

The purpose of mentoring was to support OTs in implementing ESTVR and optimise intervention fidelity and delivery. OTs delivered the intervention for up to 12-months with participants from the FRESH trial. One way of testing whether trainees have learnt a new intervention is to analyse how closely the intervention they deliver matches the intervention they were taught; also known as measuring fidelity (Hasson, 2015). Interventions with high fidelity have shown better outcomes than those with lower fidelity (Hasson, 2015). Fidelity data is not presented here as analysis remains ongoing. 
Supplementing training with ongoing support has been found successful in implementing complex therapy interventions (McCormack et al., 2013). Study design allowed four mentors (from the training group) to provide one hour of support per month per OT since this was considered typical of clinical practice in England. Mentoring was delivered flexibly and included meetings, telephone calls, emails or texts as agreed between the OT and their mentor.

Mentoring sessions were documented and supplementary data collected from emails, texts, and written summaries of phone calls between the OTs and mentors. This data will assist in the evaluation of the intervention's fidelity in the FRESH trial. The interviews with the OTs were used in evaluating the usefulness of mentoring.

\section{Interviews}

Ethical approval was gained for the interviews. OTs provided written and verbal consent to be interviewed. A topic guide (see supplementary file) was developed using a priori concepts about implementing complex interventions. The main topic areas included an introductory conversation about the OT's professional experience followed by reflections the OT had about the training they attended. Continuing a conversational style, questions then related to the perceived effectiveness of the training and experiences of putting training into practice and finally what should change to improve future training.

As the author was involved in the development and delivery of training, an independent researcher conducted the telephone interviews. An iterative process was used to reflect on data collected, and refine the topic guide for subsequent interviews. Interviews were digitally recorded, transcribed, cleaned and stored securely. Interviews occurred a mean of 4.2 months after the OTs had seen their first patient (range 2-6 months) and a mean of 7.8 months after the initial training days (range 3-12 months).

Framework approach was used to analyse interviews. This approach allows for a priori concepts (of implementation research) to act as a "framework" to explore and interpret what is happening 
in a particular setting (Ritchie, 1994). Analysis of the interviews was conducted by the author in consultation with the independent researcher who performed the interviews. NVivo 10 for Windows software was used for data management.

\section{Results}

\section{Participants}

Mentoring of OTs is ongoing; therefore results reported include the LNA, self-perceived knowledge and confidence to deliver ESTVR rating and interviews.

The training package was delivered to OTs working in the FRESH trial. Four OTs were initially recruited, two OTs in one site and an OT in each of the remaining sites. One of the original OTs dropped out and was replaced. Therefore five OTs underwent training in total, four female and one male; mean age 39.2 years (range 34 to 47 years). The OTs had been qualified a mean of 11.4 years (range 12 to 15 years). Two trained in the UK and three qualified overseas (South Africa, New Zealand and Australia). One held a higher degree in VR. All had experience of working within the NHS and working with people with neurological conditions (mean 9.7 years, range $3-15$ years).

Four out of five OTs reported having no TBI learning needs. The fifth had neurological experience with progressive conditions and identified TBI learning needs. The greatest learning needs concerned formal sick leave forms used in the UK provided by doctors (Fit Note), supplementary RTW advice that can be provided by allied health professionals (AHPs) (AHP Fitness for Work Report) and the research processes directly related to the FRESH trial. These aspects were integrated into training and additional support offered in mentoring and follow-up refresher training.

Three of the four OTs attended training at the University on the scheduled dates. The fourth therapist, who was unable to attend, received adapted training viewing video recordings before attending a one-day training at the University two months later. This OT subsequently dropped 
out and was replaced. The replacement OT's training was further adapted for pragmatic reasons and included the videos and a single day of teaching at the research site. Both of these OTs reported watching the video recordings prior to training.

Four OTs attended refresher training in April 2014 and October 2014 and the fifth received a summary of this day's events from the mentor.

All five OTs were interviewed as planned. However, one therapist left the project before recruitment commenced and never held a caseload. It is not unusual to have staff changes within the NHS and even though the OT did not implement the ESTVR it was felt appropriate to include data from the interview to gain insight into the face-to-face training experience.

\section{Self-Perceived Knowledge and Confidence}

All five OTs who completed the initial training completed self-perceived knowledge and confidence questionnaire and all four OTs who attended the refresher training six months later completed the same forms (Table 3).

Table 3. Median (range) results for self-perceived knowledge and confidence in providing ESTVR.

\begin{tabular}{|l|c|c|c|}
\hline & Pre-training & $\begin{array}{c}\text { Post- } \\
\text { training }\end{array}$ & Refresher \\
\hline Knowledge of TBI & $4(3-5)$ & $5(4-5)$ & $4(3-5)$ \\
\hline Knowledge of how TBI impacts on work & $4(3-5)$ & $4(4-5)$ & $4(3-5)$ \\
\hline Knowledge of VR in general & $4(1-4)$ & $4(3-4)$ & $3.5(3-4)$ \\
\hline How to prepare a person back to go back to work & $3(1-4)$ & $4(3-4)$ & $4(3-4)$ \\
\hline How to do a graded RTW & $3(1-4)$ & $4(3-4)$ & $4(3-4)$ \\
\hline Knowledge of research process & $2(1-3)$ & $3(2-4)$ & $4(2-4)$ \\
\hline
\end{tabular}




\begin{tabular}{|l|c|c|c|}
\hline Confidence in helping someone prepare for work & $3(1-4)$ & $4(3-5)$ & $4(4)$ \\
\hline Confidence in helping someone RTW & $3(1-4)$ & $4(3-5)$ & $4(3-4)$ \\
\hline Confidence in dealing with employers & $3(1-4)$ & $4(3-5)$ & $3.5(3-4)$ \\
\hline Confidence in working in a research project & $2(1-2)$ & $3(2-3)$ & $4(2-4)$ \\
\hline How useful was the pre-training reading & $2(3-4)$ & N/A & N/A \\
\hline How useful was the course overall? & N/A & $4(4-5)$ & $4.5(4-5)$ \\
\hline
\end{tabular}

Key: 1=poor; 2=limited; $3=$ =average; 4=good; 5=very good

The three things considered most useful directly following the initial training were: case study discussions $(n=5)$; detailed discussions about elements of the intervention $(n=3)$; and reviewing trial research processes $(n=2)$. The things considered most useful directly following the refresher day were: case study discussions $(n=4)$; revisiting the trial aims and documentation $(n=2)$; group problem-solving $(n=2)$; and reminders about manual resources $(n=2)$.

Considerations for improvements to future training included: increasing training to three days $(n=2)$; outlining the trial before the intervention $(n=2)$; using technology to improve access to learning materials before training and afterwards to build a community of practice for knowledge sharing $(n=1)$; increasing the amount of case discussion $(n=1)$; explaining key VR concepts early in the training $(n=1)$.

\section{Interview Results}

Thematic analysis of the interviews revealed six major categories of themes (Figure 1).

Figure 1. Thematic Analysis of the interviews 

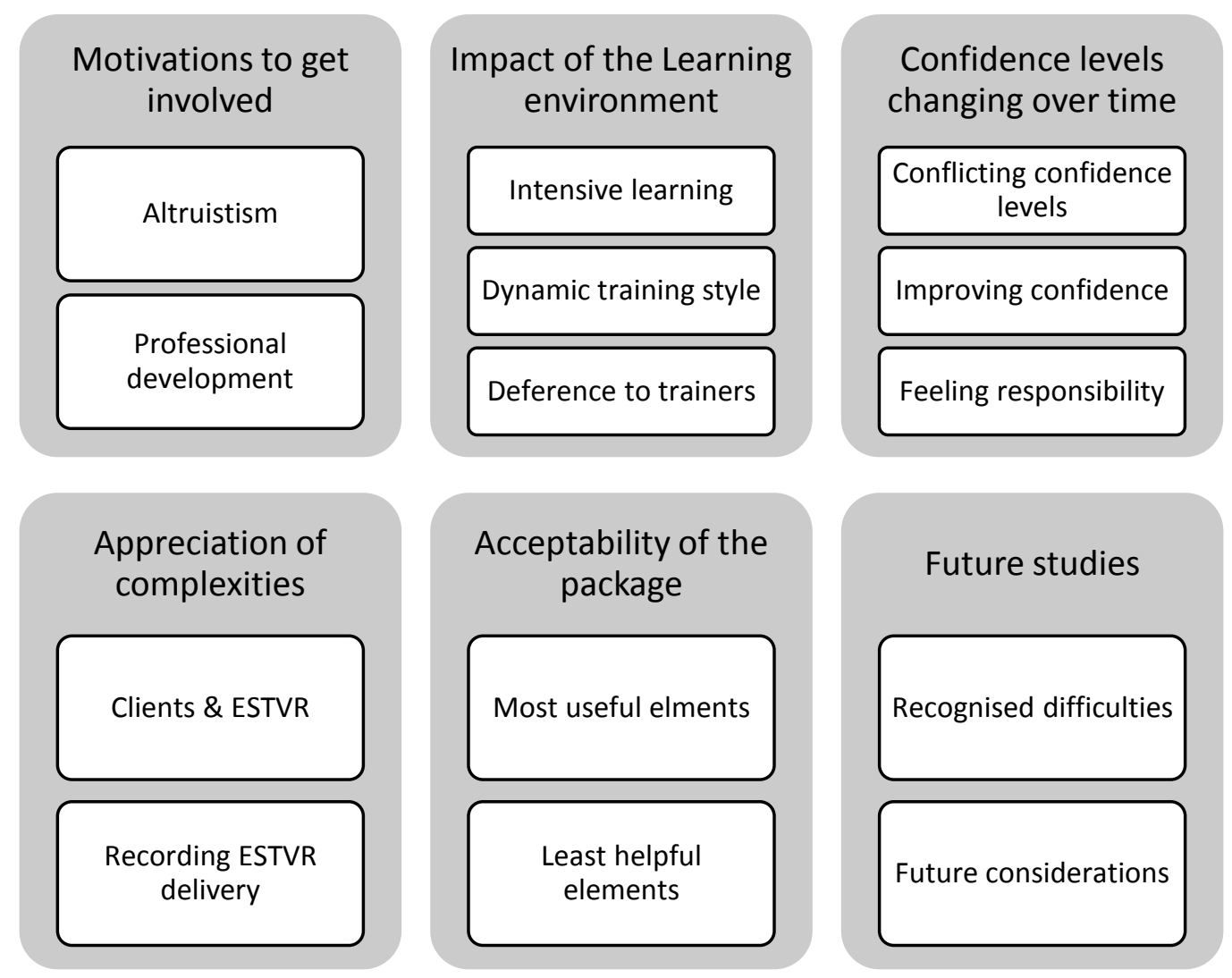

\section{Motivations to get involved}

Altruistic motivations to get involved with the trial dominated the OTs reflections: "Obviously, one, because there's not a heck of a lot of evidence in OT and VR and TBI, so anything that, I think, as a clinician, any of us can do to kind of help that evidence to be established is fantastic." (OT2). OTs also reflected that the trial would provide opportunity for professional development.

\section{Impact of the Learning environment}

When asked if the training was about the right length, they reflected that it felt intensive but: “looking at what needed to be covered, it couldn't have been done any shorter. You know, if you didn't know anything about TBI or VR, any shorter than two days, it would have been too rushed and too much information." (OT2) 
The OTs recognised the dynamic training style as flexible to meet individual needs. The flexibility facilitated communication and built confidence: "I think having access to them any time during the day to ask anything daft, or to just kind of put things in perspective, was very helpful." (OT3).

The training team was recognised for its subject expertise and this was valued: "we're being uptrained or trained by specialist OTs [named but deleted] so what's not to like about that?" (OT5). This was also mixed with some deference: "they just were standing there like these fountains of knowledge, that you could ask them anything and they would answer you." (OT4).

\section{Confidence levels changing over time}

Although the experienced OTs reported some degree of confidence prior to training, some described lacking confidence at the same time in either their skills in community work or specific clinical knowledge: "I just worry a bit about not knowing the in-depth neuro cognitive bit" (OT5). All reported improved knowledge about the intervention following training and reflected that "higher level" reasoning skills were required to individualise ESTVR: "for me, being relatively experienced, it's sort of bread and butter type stuff, just your critical reasoning is a bit higher level and you should be able to apply high-level clinical reasoning to individual clients." (OT1).

Following training one OT recognised that they had lacked depth of knowledge: "from having done the training, I think you kind of realise how much more there is to know or how many more angles there are that you didn't ever know about. And so all of a sudden you just have much more of a conscious incompetence - a sort of awareness of your incompetence more" (OT4). This highlighted a need to explore this during mentoring. Self-rated confidence scores support this reflection.

Although their knowledge improved and they had confidence in implementing ESTVR, they were conscious that their confidence was likely to increase further with experience of delivering 
the intervention: "It's still kind of a learning curve for me and I'm still finding out." (OT3). On reflection the OTs recognised that they had been unable to anticipate all their learning needs ahead of or during training and only appreciated this later once they'd had the opportunity to meet again. Some had seen participants by the time the refresher training took place, which helped this process. Discussions around implementation were based on practice rather than being theoretical: "that's where it all comes together, isn't it, when you can actually hear about how, what's written in the book translates into practice and then have an end result for a client." (OT2).

Some reported feeling under pressure to succeed but identified that that this was mostly selfimposed: "I always put pressure on myself that my interventions are going to result in something good and I always take it personally if it doesn't. I just think I'm that kind of person. I don't think the trial is putting us under any pressure at all, no. And in actual fact, they, you know, constantly reiterated they're not expecting us to be perfect." (OT2)

\section{Appreciation of complexities}

The therapists talked about the complexities surrounding the client group and tailoring the intervention being delivered: "You might think, "Okay, I can do just the same as the first one" but, no, it might look the same, it's a TBI going back to a bank but the boss might be different, the relationships are different. Everything is completely different. And, it's not as simple as saying, right, "I'll pull this tool out and I'll use the same thing." Every single one has to be different, doesn't it?" (OT2).

\section{Acceptability of the package}

All OTs reported that discussing cases during the refresher training was helpful in implementing ESTVR locally, they said they could: "discuss it [case scenario] in a group, and then learn from that and see how the Fresh process [ESTVR] actually applies to different individuals." (OT1). 
Some saw the manual was as a useful resource explaining: "I'm sure that at some point I may go back and revisit those things, depending on the clients I get, and keep go and revisit those sections as you go along." (OT4), whereas others relied heavily on it: "I kind of see it as my vocational rehab bible" (OT3) who found the written process a useful reminder. The manual was found to be useful alongside teaching and mentoring: "if I didn't have the folder and the training, I wouldn't just manage with the mentor... I think it's essential that both are in place." (OT5).

Within training sessions, service users provided opportunities for role play: "We had actual clients coming in that we can role play and practise with, which was great. I thought that was really, really good." (OT4). However, there was ambivalence about this method too: "I kind of felt that you were being assessed. That's probably more my anxiety really in that you want to try and get it right" (OT3).

Although accepted as inevitable, one of the least helpful aspects of the training was the lack of clarity and separation between learning about the intervention and learning about the trial processes: "Because it's a feasibility study, a lot of, actually the feasibility needed to be hashed out within those two days, and certainly the follow-up day, a lot of that on the follow-up day was about hashing out the processes that are happening as well as part of the study" (OT4).

Another was the time lag between training and intervention delivery: "If we went through the training in the week before and they started recruiting the week after, and in two weeks we had our first client recruited or patient recruited into the study and we were able to get straight into it, that would be an ideal situation for consolidated learning. But it was literally six months later." (OT1).

The OT who joined the study later reported that extra learning outside of work time was required to assimilate all the information: "I think the only challenge was trying to fit it all in my own time and trying to get up to speed, because as I say, I joined the study late, so I felt a bit behind and I 
was really pushing myself and my time to try and catch up with the other people that had already been on the training." (OT5).

\section{Future implementation}

When research involves a new intervention being delivered in the NHS then it is understood these excess treatment costs are the responsibility of the NHS (NHS England, 2015). Barriers associated with releasing NHS staff for trials despite secured excess treatment costs for backfill were highlighted: "They've got no leeway to release anyone, ... They just are running on such low staffing levels that they can't afford, even if they're being funded for it, they can't afford to release someone for a day a week." (OT4).

A number of considerations about future training delivery were made; the continued use of service users; flexible training , with increased prescriptiveness for therapists less experienced in TBI and or VR; an extra day focussing on describing specific research processes; minimising the delay between training and intervention delivery; greater use of case discussions and scenarios; sufficient time for familiarisation with the manual and its resources and allowing time for discussion; learning through videos was manageable only if time was specifically allocated; face-to-face learning was preferred.

\section{Discussion}

The discussion will address what has been learned through developing this learning package both from the authors' experience and from its evaluation. Then alternate procedures to improve the package including delivery of information, reminders, training to match the experience of therapists, the success of mentorship and suggestions to address the stress therapists perceived in being part of a research study.

The WIDER guidelines helped both the development of the training package and its description. Developing the package (manual, training and mentoring) was time consuming and complex. It 
involved in-depth questioning to elicit both explicit (codified, written) and tacit ("know-how") knowledge (Kothari et al., 2012) from the therapist who had delivered ESTVR over 20 years as part of NTBIS. Explicit knowledge was straightforward to access and helped guide discussions. Tacit knowledge however, was more difficult and time consuming to elicit, yet essential in describing the complexity of the intervention (Kothari et al., 2012). This required repeated discussions to achieve the level of detail needed for knowledge translation (Hoffmann et al., 2013). Whilst other studies allude to this process, few describe it in detail, which future research could address.

At the outset the OTs were expected to have relevant clinical experience of TBI but not necessarily of VR as this was the cornerstone of the training package. However, four out of the five OTs reported apriori knowledge and experience in VR and all indicated few VR related learning needs. The OT with little VR experience identified greater learning needs, which were met by the face-to-face training and through mentoring. Although the self-perceived knowledge and confidence (Table 3) indicates reduction of some scores at refresher training, the interviews indicate that confidence grew, with OTs recognising, "a learning curve" (OT3). The late start of the FRESH trial meant OTs had experienced few cases before the refresher training. This could be a reason for the scores. Future trials should aim to coordinate the commencement of participant recruitment and staff training.

Although therapy staff changes in NHS services are inevitable (O'Brien et al., 2013), contingency planning does not always occur in research. We were aware of and anticipated that it might not possible to meet our aim for all OTs to train together. Through contingency planning, video recordings and electronic copies of training materials allowed OTs who were unable to attend the original training dates to watch and listen to the presentations. Although this also had the benefit of reducing repeating face-to-face teaching, moving future training away from a face-to-face model would require caution as OTs preferred training with peers. 
Training materials were emailed to the OTs, however, some NHS computer systems didn't allow downloads or access to certain links, therefore the same was provided on memory sticks. We have no way of verifying whether the therapists reviewed the training sessions and we did not set out to evaluate their effectiveness as a training modality. However, for those considering this in future trials, the practicalities to access this technology should be considered.

The manual was valued, being seen as integral to the training package. It was developed in a way that allowed OTs to adapt it to their local context (NHS Trust and patients) thus aiding implementation. Adaptations included adding local details for other NHS rehabilitation, TBI and employer support services, referral routes, and specific assessment tools. Adaptations were also provided from the trainers such as national updates e.g. legislation. Further analysis of manual and intervention adaptations is currently underway and therefore not reported here.

The therapists valued the detailed description of the intervention in the manual describing it as a "VR bible" (OT 3). However, some indicated that they forgot about some resources between initial and refresher training and found reminders helpful. The importance of reminders has been identified by others (Graham et al., 2006) and involved case discussions and specific direction by mentors towards information e.g. driving after TBI. Future trials should allocate more time to familiarisation with the manual and to ensuring therapists understand its purpose and how to adapt it to their local context.

Despite having identified learning needs related to clinical aspects of the intervention, OTs' anxieties were not about its implementation per se but rather its delivery as part of a study and their role in the trial. Information about the design of the trial and issues that might affect outcomes e.g. contamination was included in the training but the OTs suggested more time should be spent on this in future. It is likely that therapists recruited to future trials will have some experience relevant to the clinical intervention but limited experience of research. Researchers should consider how best to convey study details to assimilate this new learning. 
In particular addressing how delivering the intervention in a trial might differ from usual clinical practice. Mentors can reinforce information and help problem-solve trial related issues, thus preventing them from becoming implementation barriers, involving and informing the Chief Investigator throughout. This approach was used in this study.

Learning new information and putting this into practice requires assimilation before competence is achieved. Supporting OTs' knowledge and skill development to deliver ESTVR was factored into the design of the training package by the inclusion of mentoring. Mentoring was designed as a peer-to-peer platform to share knowledge and problem-solve local implementation issues. It was also intended as a mechanism for ensuring fidelity and optimising participant outcomes. However, the extent to which it has been successful in achieving these outcomes or in affecting the competency and skills of the therapists remains unclear and an area for further research. An hour per month of telephone support was factored into the study design but a more flexible interpretation meant face-to-face contact, email and texts were also used. The OTs predicted that they would become more confident with experience and as expected the intensity of mentoring reduced over the lifetime of the study. Further analysis of the mentoring data may reveal whether OTs were supported to overcome implementation barriers and how much time this took.

\section{Study limitations}

Findings report on the experiences of five OTs participating in a multicentre trial and are unlikely to be representative of all clinical OTs, or those from other professional backgrounds trained in other studies. However, the findings highlight some useful points for consideration when developing training relevant to trial interventions. As there was no training for therapists in the comparator arm it remains unclear whether an alternative training package might have been equally acceptable. 


\section{Conclusions}

Qualitative evaluation of the training package suggests the OTs absorbed the knowledge necessary to implement ESTVR within the trial and knowledge transfer was successful. Therapists found the training package acceptable and useful and highlighted important factors for consideration in future trials. Future delivery would also consider additional measures of its effectiveness in knowledge transfer.

\section{Key Messages}

- Training packages require detailed descriptions of interventions to enable implementation and adequate resources should be allocated.

- Therapists have concerns about implementing interventions as part of research that should be addressed.

This study helps OTs to understand components of a VR training package for people with TBI and shows OTs' positive experiences of the training prepared them in implementing the intervention.

\section{References:}

Albrecht L, Archibald M, Arseneau D, et al. (2013) Development of a checklist to assess the quality of reporting of knowledge translation interventions using the Workgroup for Intervention Development and Evaluation Research (WIDER) recommendations. Implement Sci 8: 52.

Black C and Frost D. (2011) Health at work - an independent review of sickness absence. London: The Stationary Office.

Cancelliere C, Cassidy J and Colantonio A. (2013) Specific disorder-linked determinants: traumatic brain injury. In: Loisel P and JR A (eds) Handbook of work disability: prevention and management. . 1 ed. New York: Springer-Verlag New York, 303-314.

Cancelliere C, Kristman VL, Cassidy JD, et al. (2014) Systematic review of return to work after mild traumatic brain injury: results of the international collaboration on mild traumatic brain injury prognosis. Arch Phys Med Rehabil 95: S201-209.

College of Occupational Therapists. (no date-a) Advice on how to overcome work-related stress. . Available at: https://www.cot.co.uk/sites/default/files/marketing materials/public/work-relatedstress-leaflet.pdf. 
College of Occupational Therapists. (no date-b) Working is good for you. Available at: https://www.cot.co.uk/sites/default/files/marketing materials/public/working-isgood-for-you.pdf.

Damschroder L, Aron D, Keith R, et al. (2009) Fostering implementation of health services research findings into practice: a consolidated framework for advancing implementation science. Implement Sci 4: 50.

das Nair R, Lincoln N, Phillips C, et al. (2012) Rehabilitation of Memory following Brain Injury a phase III Randomised Controlled Trial University of Nottingham: National Institute of Health Research - Health Technology Assessment.

Department for Work and Pensions. (2013) 2010 to 2015 government policy: employment. London. London: The Stationary Office.

Department of Health. (2010) The NHS Outcomes Framework 2011/12. London. The Stationary Office.

Escorpizo R, Finger M, Glässel A, et al. (2011) An International Expert Survey on Functioning in Vocational Rehabilitation Using the International Classification of Functioning, Disability and Health. Journal of Occupational Rehabilitation 21: 147-155.

Fadyl J and McPherson K. (2009) Approaches to vocational rehabilitation after traumatic brain injury: a review of the evidence. J Head Trauma Rehabil. 24: 195-212.

Garfield A, Holmes J, Ford E, et al. (2013) Low mood and MS - a group adjustment course. University of Nottingham.

Graham I, Logan J, Harrison M, et al. (2006) Lost in knowledge translation: time for a map? J Contin Educ Health Prof. 26: 13-24.

Hasson H. (2015) Intervention fidelity in clinical trials. In: Richards DA HI (ed) Complex interventions in health: an overview of research methods. Abingdon, Oxon: Routledge, 232-238.

Hoffmann T, Erueti C and Glasziou P. (2013) Poor description of non-pharmacological interventions: analysis of consecutive sample of randomised trials. BMJ 347: $\mathrm{f3755}$.

Kothari A, Rudman D, Dobbins M, et al. (2012) The use of tacit and explicit knowledge in public health: a qualitative study. Implement Sci 7: 20.

Logan P, Armstrong S, Avery T, et al. (2014) Rehabilitation aimed at improving outdoor mobility for people after stroke: a multicentre randomised controlled study (the Getting out of the house study) Health Technology Assessment.

McCormack B, Rycroft-Malone J, DeCorby K, et al. (2013) A realist review of interventions and strategies to promote evidence-informed healthcare: a focus on change agency. Implement Sci 8: 107.

Medical Research Council. (2008) Developing and evaluating complex interventions: new guidance: London: Medical Research Council.

NHS England. (2015) Guidance on Excess Treatment Costs. In: England N (ed). NHS England, London.

O'Brien R, Woodbridge S, Hammond A, et al. (2013) The Development and Evaluation of a Vocational Rehabilitation Training Programme for Rheumatology Occupational Therapists. Musculoskeletal Care 11: 99-105.

Petzold A, Korner-Bitensky N and Menon A. (2010) Using the knowledge to action process model to incite clinical change. J Contin Educ Health Prof 30: 161-171. 
Phillips J. (2013) Return to work after traumatic brain injury: a cohort comparison study and feasibility economic analysis. Rehab and Ageing. University of Nottingham.

Phillips J, Drummond A, Radford K, et al. (2010) Return to work after traumatic brain injury: recording, measuring and describing occupational therapy intervention. British Journal of Occupational Therapy 73: 422-430.

Playford D and Sweetland J. (2010) Working yet worried. MS Society.

Playford E, Radford K, Burton C, et al. (2011) Mapping Vocational Rehabilitation Services for people with Long term neurological conditions: Summary report. Department of Health.

Radford K, Phillips J, Drummond A, et al. (2013a) Return to work after traumatic brain injury: Cohort comparison and economic evaluation. Brain Injury 27: 507-520.

Radford K, Watkins C, Sutton C, et al. (2013b) FRESH - Facilitating Return To Work Through Early Specialist Health-Based Interventions. NIHR HTA Programme

Richards DA. (2015) The complex interventions framework. In: Richards DA HI (ed) Complex interventions in health: an overview of research methods. Abingdon, Oxon: Routledge, 1-15.

Ritchie JS, L. . ( 1994) Qualitative data analysis for applied policy research. In: Bryman A BR (ed) Analyzing qualitative data. 173- 194.

Saltychev M, Eskola M, Tenovuo O, et al. (2013) Return to work after traumatic brain injury: Systematic review. Brain Inj 27: 1516-1527.

The Royal College of Physicians and The British Society of Rehabilitation Medicine. (2003) Rehabilitation following acquired brain injury: National clinical guidelines. London: RCP, BSRM.

Walker M, Sunderland A, Fletcher-Smith J, et al. (2012) The DRESS trial: a feasibility randomized controlled trial of a neuropsychological approach to dressing therapy for stroke inpatients Clinical Rehabilitation 26: 675-685. 